\title{
Ein optisch-inaktives Natriumsalz der Nucleinsäure.
}

Von

R. Feulgen.

(Aus dem physiologischen Institut der Universităt Berlin.)

(Der Redalition zugegangen am 22. September 1918.)

Mit zwei Kurvenzeichnungen im Text.

Beim Ausfällen von thymonucleinsaurem Natrium mit Alkohol aus wässeriger, mit Natronlauge alkalisch gemachter Lösung fiel mir auf, da $\beta$ der abgeschiedene Körper gegen Lackmus und Phenolphthalein stark alkalisch reagierte und einen erheblich erhöhten Aschengehalt aufwies auch dann, wenn er darauf zu wiederholten Malen aus reinem Wasser umgefällt wurde. Da Natriumhydroxyd in der aus verdünntem Alkohol bestehenden Mutterlauge leicht löslich ist, und das bisher bekannte 4 basische nucleinsaure Natrium ein gegen Indikatoren neutral reagierender Stoff ist, so wurde diese Eigentümlichkeit weiter untersucht. Es stellte sich die auffällige Tatsache heraus, $\mathrm{da} B$ die alkalisch reagierende Substanz nunmehr völlig in aktiv war, während das nucleinsaure Natrium vordem (in neutraler Lösung) die ansehnliche spezifische Drehung von $80^{\circ}$ aufwies. Ich stelle hier gleich ausdrücklich fest, daß nach weiterem Zusatz von Natronlauge sich der optisch-inaktive Zustand nicht änderte und insbesondere keine Neigung zeigte, in eine Linksdrehung überzugehen.

Die weitere Untersuchung ließ erkennen, da $\mathrm{B}$ die $\mathrm{Ab}$ nahme der Drehkraft eine gleichmäßige war, und zwar verringert sie sich proportional der Menge des zugesetzten Natriumbydroxyds bis zum Nullwerte. Eine bleibende Veränderung des nucleinsauren Natriums war insofern nicht eingetreten, als die

Hoppe-Seyler's Zeitschrift f. physiol. Chemie. CrV. 
alte Drehung sofort wieder auftrat, wenn die alkalische Reaktion mit einer Säure wieder rückgängig gemacht wurde. Dieses Spiel wurde ohne Zersetzung der Nucleinsäure beliebig oft wiederholt.

Eine merkliche Dauer des Verlaufs dieses Vorganges konnte nicht ermittelt werden, vielmehr war der Prozeß bereits vollzogen, wenn das Beobachtungsrohr beschickt und in den Polarisationsapparat eingelegt worden war. Erscheinungen vom Charakter der Multirotation wurden niemals beobachtet.

a- und b-nucleinsaures Natrium verhielten sich bei diesen Versuchen vollkommen gleich. Bei a-nucleinsaurem Natrium ergab sich aber eine wichtige Besonderheit. Mit dem Verschwinden der optischen Aktivität war nämlich hierbei auch ein Verschwinden der Gelatinierfähigkeit verbunden. Diese trat aber ebenfalls wieder auf, wenn nach Neutralisation der alkalischen Reaktion die optische Aktivität wiederhergestellt wurde.

$\mathrm{Da} B$ Natronlauge imstande ist, die Gelatinierfähigkeit des a-nucleinsauren Natriums aufzuheben, ist eine seit langem bekannte Tatsache. Eine Erklärung war aber bis jetzt nicht möglich. Worauf es hier ankommt, ist die enge Beziehung zwischen Gelatinierfähigkeit und optischer Drehung, eine $A b-$ hängigkeit, für die im Laufe dieser Arbeit noch weitere Beispiele mit anderen Eingriffen angeführt werden sollen. Bei b-nucleinsaurem Natrium kann eine solche Abhängigkeit deswegen nicht bestehen, weil b-nucleinsaures Natrium überhaupt nicht gelatiniert.

Eine gewisse Abhängigkeit der Größe der optischen Drehung von der Reaktion ist schon von Walter Jones ${ }^{1}$ ) beobachtet worden. Dieser Autor wandte zur Hervorrufung der alkalischen Reaktion Ammoniak an. Eine völlige Inaktivierung konnte er aber - aus später zu erkennenden Gründen - nicht beobachten. Es kam ihm auch weniger darauf an, eine Verringerung der Drehkraft nachzuweisen, als vielmehr zu zeigen, daß auch Nucleinsäuren anderer Herkunft sich gleich verhielten, woraus er auf eine. Identität der Nucleinsäuren

1) W. Jones, Journ. of Biol. Chem., Bd. 5, S. 11 (1908). 
schloß, eine Folgerung, die in diesem Ausmaße schon aus dem Grunde unberechtigt ist, weil z. B. Thyminsäure sich ebenso verhält. Jones hat jedoch . nicht die Abhängigkeit der Drehung von der Reaktion auf eine einfache Beziehung zurückgeführt, was auch, wie weiter unten auseinandergesetzt werden wird, unmöglich ist, wenn man zur Herbeiführung der alkalischen Reaktion Ammoniak verwendet.

Eine nur quantitative Änderung des optischen Drehvermögens würde keine auffällige Erscheinung sein, sind doch optisch aktive Säuren bekannt, die als Alkalisalze sogar eine entgegengesetzte Drehrichtung zeigen. In diesen Fällen bleibt der Körper optisch aktiv. Hier handelt es sich aber, und das muß mit Nachdruck betont werden, um ein vollkommenes Verschwinden jeglicher optischen Aktivität, und der Nullwert der Drehung ist nicht etwa ein Übergangsstadium in die entgegengesetzte Drehrichtung oder eine Zufallserscheinung; denn es konnte auch mit einem vollkommenen Lippichschen Halbschattenapparate keine Spur von Drehung mehr beobachtet werden, und während gewöhnliches, aktives, nucleinsaures Natrium hinsichtlich seiner Drehkraft eine große Abhängigkeit von der Konzentration und eine sehr erhebliche Abhängigkeit von der Temperatur aufweist, blieb einmal inaktiviertes nucleinsaures Natrium auch bei verschiedenen Temperaturen und verschiedenen Konzentrationen völlig inaktiv.

Aus den wenigen bisher angeführten Tatsachen geht wohl schon hervor, daß das Natriumhydroxyd mit dem nucleinsauren Natrium eine Salzbindung eingegangen ist, da $\$$ dieses Salz optisch inaktiv ist, und daß damit weitere Säuregruppen oder als Säure wirkende Gruppen aufgefunden worden sind, die von ganz besonderer Art sein müssen. Diese Säuregruppen sind sehr schwach; denn die Natriumsalze derselben zeigen weitgehende hydrolytische Dissoziation, und das inaktive nucleinsaure Natrium reagiert deswegen stark alkalisch. In der Tat konnten diese schwachen Säuregruppen mit Indikatoren nicht nachgewiesen werden.

Es gibt nun eine überraschend einfache Methode, die „Stärke ${ }^{*}$ dieser Säuregruppen abzuschätzen: Eine beliebige 
(inaktive) Säure, die man zu inaktiviertem nucleinsauren Natrium hinzusetzt, hebt dann den Salzzustand dieser schwachen Säuregruppe auf, wenn sie "stärker" ist als diese. Die Aufhebung des Salzzustandes erkennt man aber bequem an dem Wiederauftreten der Drehung. Man hat also nur nötig, das nucleinsaure Natrium mit Natronlauge eben zu inaktivieren und eine äquivalente Menge der zu vergleichenden Säure zuzusetzen. Tritt Drehung auf, so muß die vergleichende Säure "stärker" sein als die Säuregruppen in dem nucleinsauren Natrium.

Derartige Versuche haben nun ergeben, daß die neuen Säuregruppen nicht nur schwächer als die Essigsäure (die von der Phosphorsäure herrührenden Säuregruppen sind stärker als Essigsäure), sondern auch schwächer als die Kohlensäure sind, ja sogar schwächer als der II. Dissoziationsgrad in derselben; denn Einleiten von Kohlendioxyd oder Zusatz von Natriumbicarbonat stellen die Drehung wieder her, während chemisch neutral gebaute Salze, z. B. Natriumchlorid, natürlich keinerlei Einfluß auf den inaktiven Zustand des inaktivierten nucleinsauren Natriums ausüben. $\mathrm{Da}$ auch das Phenol inıstande ist, den Salzzustand aufzuheben, so ist die Stärke der neuen Säuregruppen in dem nucleinsauren Natrium schwächer als der Säurecharakter des Phenols. Der Wert der Dissoziationskonstante wird demnach unter $5,8 \cdot 10{ }^{11}$ liegen.

In Anbetracht des geringen Dissoziationsgrades der Säuregruppen ist es nicht verwunderlich, daß schwächere Basen keine beständigen Salze mehr bilden können. Gibt man zu inaktiviertem nucleinsauren Natrium die äquivalente Menge Ammoniumchlorid, so tritt Drehung wieder fastim alten Betrage auf, weil die Säuregruppen unter Bildung von Ammoniak und Natriumchlorid frei werden. Erst ein großer Überschuß von Ammoniak läßt die spezifische Drehung sinken (vgl. Jones). Die Ammoniumsalze der Säuregruppen neigen also zum hydrolytischen Zerfall.

-Von Wichtigkeit ist nun die Frage nach dem Orte dieser Säuregruppen, besonders wenn man berücksichtigt, daß Levene in seiner Nucleinsäureformel ${ }^{1}$ ) eine durch die Phosphorsäure

1) Journ. of Biol. Chem., Bd. 12, S. 417. 
hervorgerufene sechsbasische Natur der Nucleinsäure voraussetzt, während bis jetzt nur ein vierbasischer Charakter bewiesen ist. Sollten die neu aufgefundenen Säuregruppen mit den von Levene geforderten, aber noch nicht nachgewiesenen überzähligen Phosphorsäurevalenzen identisch sein? Ich halte dies für sehr unwahrscheinlich.

Zunächst ist die große Schwäche der neuen Säuregruppen etwas, was gegen die Wahrscheinlichkeit spricht, daß3 die Säuregruppen durch Phosphorsäure hervorgerufen werden; denn überall da, wo uns im Bereiçhe der Nucleinsäure und ihrer Derivate Phosphorsäuregruppen entgegentreten, sind sie von so großer Stärke, daß sie beständige Baryumund sogar beständige Brucinsalze bilden können. So stellte Levene ein vierbasisches Brucinsalz seiner „Cytosin-Hexose-

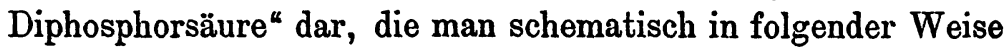
zum Ausdruck bringen kann:

$$
\begin{aligned}
& =\text { Phosphorsäure } \\
& =\text { Phosphorsäure }
\end{aligned} \text { Kohlenhydrat }- \text { Cytosin. }
$$

Ferner spricht gegen die Annahme von Phosphorsäuregruppen die so leicht reversible innere Umlagerung, die sich bei der Salzbildung im Molekül desjenigen Komplexes vollzieht, der allein für die optische Aktivität verantwortlich zu machen ist: nämlich im kohlenhydratähnlichen Körper. Dieser Vorgang spricht eher für die Beteiligung einer organischen als einer Phosphorsäuregruppe.

Es mußte nun weiter die Frage beantwortet werden, ob etwa die in der Nucleinsäure vorhandenen Basen an dem Vorgange der Inaktivierung beteiligt sind. Dies konnte, soweit diePurinbasen in Betracht kommen, experimentell in verneinendem Sinne entschieden werden; denn die Thyminsäure wurde ebenso in alkalischer Lösung inaktiv.

Präparative Darstellung des inaktiven nucleinsauren Natriums.

Man löst nucleinsaures Natrium zu etwa $5-10 \%$ in Wasser, gibt etwa vier Moleküle Natronlauge hinzu und ver- 
mischt mit 3 Volumina Alkohol. Es entsteht eine milchige Trübung, die nach Zusatz von etwas konzentrierter Natriumacetatlösung als inaktives Natriumsalz der Nucleinsäure ausgeflnckt wird. Das inaktive a-nucleinsaure Natrium fällt bei genulgendem Alkoholzusatz flockig, das inaktive b-nucleinsaure Natrium aber stets sirupartig aus. Nach dem Absitzen entwässert man die Niederschläge mit Alkohol und trocknet im Exsikkator.

Eigenschaften.

Das inaktive nucleinsaure Natrium stellt ein weißes, alkalisch reagierendes, bei feuchter Luft sehr hygroskopisches Pulver dar, das in Wasser viel leichter löslich ist als die aktiven Formen. In allen anderen Lösungsmitteln hingegen ist es unlöslich. Frisch bereitet gelatiniert eine wässerige Lösung von inaktivem a-nucleinsauren Natrium nicht, auch nicht in hoher Konzentration oder in Gegenwart von Salzen (Natriumacetat). Beim längeren Stehen an der Luft (über Nacht) wird jedoch Kohlensäure angezogen, wodurch die schwachen Säuregruppen frei werden, und sowohl Gelatinierung als auch optische Drehung allmählich wieder auftreten. Die inaktiven Salze der Nucleinsäuren sind mit Alkohol viel schwieriger zu fällen als die gewöhnlichen aktiven Formen. Der Geschmack ist ziemlich stark, aber rein alkalisch, etwa wie Soda (gewöhnliches nucleinsaures Natrium ist geschmacklos).

Über die Anzahl der vorhandenen Säuregruppen.

Weiter mußte die Frage nach der Anzahl der neuen Säuregruppen untersucht werden. Hier ergab sich zunächst, daß etwa Natriumbestimmungen in dem inaktiven nucleinsauren Natrium keinen befriedigenden Aufschluß geben können. Denn einerseits hat das nucleinsaure Natrium ein hohes Molekulargewicht (ca. 1400), und es sind bereits im vierbasischen Salze 4 Atome Natrium vorhanden. Dann aber ist das nucleinsaure Natrium amorph, die Analysenzahlen sind deswegen nicht in dem Maße auszunutzen wie bei kristallisierten Körpern, nnd endlich enthalten die Präparate stets unkontrollierbare Mengen fremder Aschenbestandteile, die die Natriumbestim- 
mungen in hohem Grade fälschen können, besonders wenn man berücksichtigt, daß nucleinsaures Natrium zum Ausflocken mit Alkohol immer der Gegenwart von Salzen (Natriumacetat) bedarf.

Aus diesen Gründen wurden andere Methoden angewandt. Es wurde zunächst versucht, Klarheit über den Verlauf der Inaktivierung $z u$ verschaffen. In einer Reihe von Versuchen wurden die spezifischen Drehungen des nucleinsauren Natriums bei konstanter Temperatur und in konstanter Konzentration, aber mit wechselndem Alkaligehalte beobachtet, und zwar wurden die zugesetzten Alkalimengen von $1 / 2$ zu $1 / 2$ Molekül $\mathrm{NaOH}$ (auf ein Molekül nucleinsaures Natrium gerechnet) abgestuft. In den Versuchen wurden je $1 \mathrm{~g}$ nucleinsaures Natrium (von lufttrockenem entsprechend mehr) in einem $10 \mathrm{ccm}$ fassenden Meßkölbchen in etwas Wasser gelöst, die berechnete Menge Natriumbydroxyd in Form von $\mathrm{n} / 10^{-}$oder $\mathrm{n} / 2$-Natronlauge zugefügt, bis zur Marke aufgefüllt, und im Polarisationsapparate beobachtet. Es wurde mithin eine "Titration" ausgeführt mit der optischen Drehung als „Indikator“.

Das benutzte nucleinsaure Natrium war nicht völlig neutral, weder gegen Indikatoren, noch hinsichtlich des Verhaltens der spezifischen Drehung; denn es konnte etwa $1 / 4$ Molekül $\mathrm{NaOH}$ zugesetzt werden, ohne daß die spezifische Drehung abnahm. Die beobachteten Werte waren folgende $(c=10$; $1=1 ; t=21^{\circ}$ ):

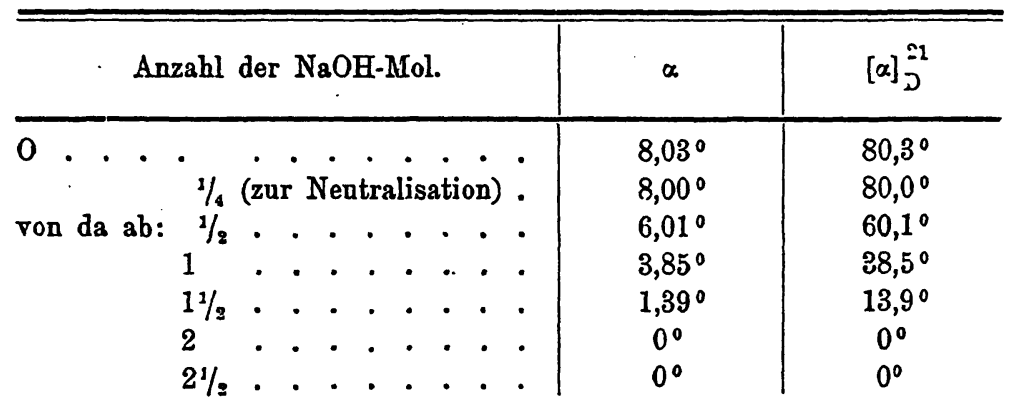

Bringt man diese Werte in einer Kurve zum Ausdruck, deren Abszissen die Anzahl der Moleküle NaOH (auf ein Molekül nucl. Natr. gerechnet), deren Ordinaten die spezifischen 
Drehungen bei den entsprechenden Alkalimengen darstellen, so erhält man das Bild der Figur 1.

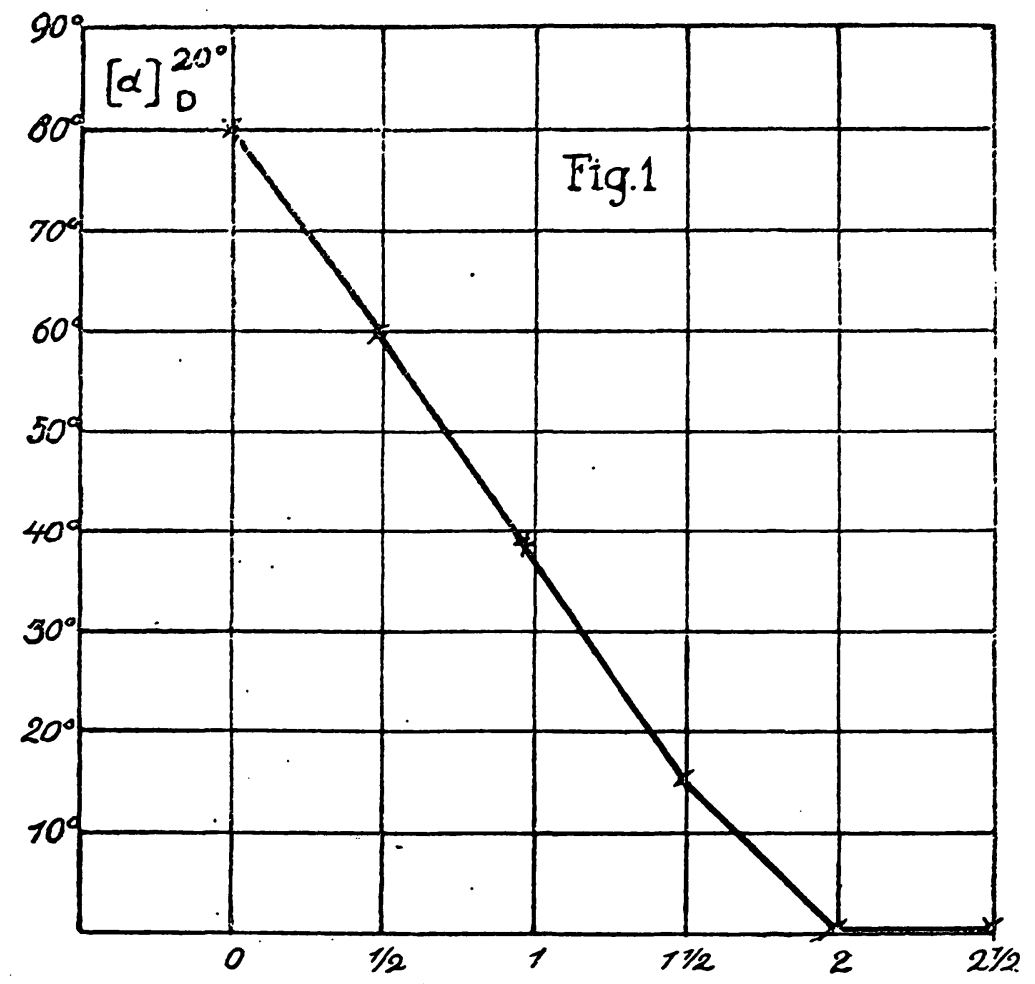

Das erste wichtige Ergebnis der Kurve ist der geradlinige Verlauf derselben, der anzeigt, daß der Prozeß ein proportionaler ist und mithin den Charakter einer Salzbildung trägt. Im hohen Grade auffällig aber muß die Tatsache sein, daß 2 Moleküle $\mathrm{NaOH}$ zur optischen Inaktivierung unter Salzbildung ausreichen, während doch im ganzen vier kohlenhydratähnliche Komplexe in der Nucleinsäure vorhanden sind. Bevor man aber aus dieser Erscheinung die Konsequenzen zieht, mußte man auf andere Weise den Gehalt an eingetretenen Na-Atomen ermitteln.

Ich stellte mir inaktives nucleinsaures Natrium dar, und zwar wurde diesmal zur Lösung des nucleinsauren Natriums ein erheblicher Überschuß von $\mathrm{NaOH}$ zugesetzt (6-8 Moleküle). 
Nach der Fällung mit Alkohol überzeugte ich mich davon, ob auch nicht etwa ein hydrolytischer Zerfall der Salze, der einen zu geringen $\mathrm{Na}-\mathrm{Gehalt}$ vortäuschen könnte, eingetreten war. Da der Körper noch optisch inaktiv war, so entfiel dieser Verdacht. Nun wurde der alkalisch reagierende Körper mit $\mathrm{n} / 10^{-}$-Säure zurücktitriert unter Verwendung der verschiedensten Indikatoren. Die erzielten Resultate waren nicht scharf, weil nach der Neutralisation des alkalisch reagierenden Salzes nicht etwa freie Titriersäure auftritt, die einen scharfen Indikatorumschlag bewirken könnte, sondern die viel schwächere freie Nucleinsäure. Versuche an gewöhnlichem nucleinsauren Natrium unter Hin- und Hertitrieren ergaben, daß man bei derartigen Titrationen mit einer Fehlerquelle von $\pm 0,4$ Moleküle $\mathrm{NaOH}$ rechnen muß. In dieser Weise wurden nun etwa ein Dutzend Titrationen von inaktivem nucleinsauren Natrium ausgeführt, aber niemals wurde das Vorhandensein von weiteren 4 Natriumatomen festgestellt, vielmehr deuteten alle Versuche auch hier darauf hin, daß nur 2 von den schwachen Säuregruppen vorhanden sind. Ich lasse hier einige dieser Versuche folgen:

$$
\begin{aligned}
& 0,4950 \mathrm{~g} \text { entsprachen } 7,6 \mathrm{ccm} \mathrm{n} / 10^{-S a ̈ u r e ~(I n d i k a t o r ~ P h e n o l p h t h a l e i n) ~} \\
& 0,6622, \quad 9,6, n / 10,(n \text { Lackmus) } \\
& 0,1988 \text { \# } 3,2, n / 10 \text { ( \# Methylorange) } \\
& \text { Gefunden: } 2,2 ; 2,08 ; 2,3 \text { Atome } \mathrm{Na} \text {. }
\end{aligned}
$$

Man muß also mit der Tatsache rechnen, daß in der Tat zwei Moleküle Natriumhydroxyd imstande sind, das ganze Molekül der Nucleinsäure zu inaktivieren, obgleich von den Komplexen, die allein die optische Aktivität bedingen können, im ganzen vier vorhanden sind. Ich kann nicht umhin zu betonen, da $B$ dieses Ergebnis eine schwerwiegende Komplikation in sich schließt, welche die Aufklärung des kohlenhydratähnlichen Körpers in der Nucleinsäure sehr behindert; denn man muß mit der Möglichkeit rechnen, daß in der Nucleinsäure $\mathrm{zwei}$ verschiedene kohlenhydratähnliche Komplexe vorhanden sind, von denen die einen beiden die beschriebene Eigentümlichkeit zeigen, unter Salzbildung inaktiv zu werden, während die anderen beiden überhaupt optisch inaktiv sind, oder doch inaktiv erscheinen. Diese Vorstellung findet eine 
Stütze in der Tatsache, daß die Purinnucleotide sich in Wirklichkeit ganz anders verhalten als die Pyrimidinnucleotide; wird z. B. Nucleinsäure mit $2 \%$ iger Schwefelsäure gekocht, so sind die Pyrimidinnucleotide noch nach $z$ weistündiger Kochdauer unversehrt (Levene), während die Purinnucleotide bereits in ebensoviel Minuten ihre Purinbasen verlieren und weiterhin zerstört werden. Eine weitere wesentliche Klärung dieser Frage wird man von dem Verhalten der von Levene isolierten Cytosin-Hexose-Diphosphorsäure und ihrer Verwandten, der Thymin-Hexose-Diphosphorsäure, erwarten. Levene gibt für diese nucleotidähnlichen Körper Rechtsdrehung an. Über ihr optisches Verhalten zu Alkalien werde ich Versuche anstellen.

Wie -soll man sich den Vorgang der Inaktivierung erklären? Unter der Voraussetzung, daß sich diese Erscheinung nur an zwei kohlenhydratähnlichen Komplexen abspielt, könnte man geneigt sein, den Vorgang etwa folgendermaßen darzustellen:

In dem gewöhnlichen vierbasischen nucleinsauren Natrium sind an zwei von den kohlenhydratähnlichen Komplexen je eine Säuregruppe oder als Säure wirkende Gruppe vorhanden. Sind diese im Säurezustande, also wegen ihrer großen Schwäche nicht elektrolytisch dissoziiert, so ist das nucleinsaure Natrium aktiv und gelatiniert in seiner a-Form. Sind sie aber als Alkalisalze vollkommen dissoziiert, so tritt zugleich eine Umlagerung in den kohlenhydratähnlichen Komplexen ein, mithin ist das elektrolytisch vollständig dissoziierte Salz, d. h. das Ion, optisch inaktiv, und die innere Umlagerung kennzeichnet sich in einer Änderung einer weiteren physikalischen Eigenschaft: Das inaktive a-nucleinsaure Natrium gelatiniert nicht mehr. Ein wesentlicher Unterschied der beiden Modifikationen bestünde dann darin, daß die Säuregruppen in der aktiven Form nicht elektrolytisch dissoziiert, in der inaktiven Modifikation aber vollkommen dissoziiert vorkommen.

Ich habe nun aber eine Reihe von Erscheinungen beobachtet, die darauf hindeuten, daß der Dissoziationsgrad der 
Säuregruppen garnicht das Wesentliche ist, daß vielmehr jene Umwandlung, die sich in der Veränderung der optischen Drehkraft und der Gelatinierfähigkeit kundtut, auch eintreten kann, wenn die Säuregruppen sich in nicht dissoziiertem Zustande befinden. Da dieser Umstand für die Auffassung des ganzen Vorganges von grundlegender Bedeutung ist, so sollen im folgenden diese Erscheinungen besprochen werden.

\section{Die Herabsetzung der Gelatinierfähigkeit und der optischen Drehkraft auch durch Säuren.}

Es ist eine seit langem bekannte Tatsache, daß auch Essigsäure die Gelatinierfähigkeit des a-nucleinsauren Natriums herabsetzen kann. Da ich nun eine enge Beziehung zwischen Gelatinierfähigkeit und optischer Drehung nachgewiesen habe, so lag die Vermutung nahe, daß durch Essigsäure auch die spezifische Drehung des nucleinsauren Natriums herabgesetzt werden könne. Dies ist in der Tat der Fall, wie folgender. Versuch lehrt:

Nucleinsaures Natrium wurde zu gleichen Konzentrationen einmal in Wasser, das andere Mal in 10\% iger Essigsäure gelöst, die optischen Werte waren folgende: $c=8 ; 1=1$; $\mathrm{t}=22^{\circ}$.

$0,800 \mathrm{~g}$ in Wasser zu $10 \mathrm{ccm}$ gelöst; $\alpha=4,39^{\circ}$

0,800 , , $10 \%$ iger Essigsäure zu $10 \mathrm{ccm}$ gelöst $\alpha=2.39^{\circ}$

$[a]_{\mathrm{D}}^{22^{\circ}}$ in Wasser $=58,0^{\circ}$; in Essigsäure $=30,0^{\circ}$.

Durch Essigsäure wird also die spezifische Drehung auf die Hälfte reduziert. Dementsprechend wurde auch eine vollkommene Aufhebung der Gelatinierfähigkeit nicht beobachtet, sondern nur eine Herabsetzung. Hat das verwendete a-nucleinsaure Natrium wegen größtenteils erfolgten Abbaus in die b-Modifikation einen Gallertschmelzpunkt, der nicht sehr wesentlich über Zimmertemperatur liegt, so kann durch Zusatz von Essigsäure eine vollkommene Aufhebung der Gelatinierfähigkeit vorgetäuscht werden.

Der Versuch mit Essigsäure steht aber mit der obẹn 
entwickelten Theorie des Vorganges in Widerspruch. Essigsäure ist ein Elektrolyt mit gleichem Ion (H-Ion) wie die Säuregruppen. Mithin muß Essigsäure den Dissoziationsgrad der Säuregruppen verringern, und die spezifische Drehung sowie die Gelatinierfähigkeit müßten zunehmen. Wenn nun auch wegen des ohnehin sehr geringen Dissoziationsgrades der schwachen Süuregruppen dies am optischen Verhalten kaum festzustellen wäre, so dürfte doch auf keinen Fall eine starke Herabsetzung der Rotation und Gelatinierfähigkeit platzoreifen. Da diese aber doch beobachtet worden sind, so beweist der Versuch, daß eine Herabsetzung der Aktivität und der Gelatinierfähigkeit auch bei freien, nicht dissoziierten Säuregruppen möglich ist.

II. Die Erhöhung der Gelatinierfähigkeit und spezifischen Drehung durch Salze (Natriumacetat).

Es ist ferner eine seit langem bekannte Erscheinung, daß Salze (Natriumacetat) imstande sind, die Gelatinierfähigkeit des a-nucleinsauren Natriums zu erhöhen. Versuche, die ich in dieser Richtung angestellt habe, zeigten, daß solche Salze, die wegen weitgehender hydrolytischer Dissoziation $\mathrm{OH}-$ Ionon abspalten, die Gelatinierfähigkeit herabsetzen können, z. B. Natriumcarbonat. Frühere Versuche ${ }^{1}$ ) ergaben z. B. den Gallertschmelzpunkt einer 5\% igen Gallerte in Wasser zu $60^{\circ}$, in n-Natriumcarbonatlösung zu $56^{\circ}$. Eine Erklärung ergibt sich sofort aus der Möglichkeit einer, wenn auch nur geringen Salzbildung infolge Vorhandenseins der $\mathrm{OH}-I$-nen. Neutral sich verhaltende Salze, oder solche Salze, die nur verhältnismäßig sehr wenig hydrolytisch dissoziiert sind, wie z. B. Natriumacetat, setzen den Gallertschmelzpunkt sehr erheblich herauf. Durch die Gegenwart von $20 \%$ Natriumacetat wurde z. B. der Gallertschmelzpunkt einer $5 \%$ igen Gallerte um $17^{\circ}$ erhöht. Entsprechend der Abhängigkeit der Gelatinierfähigkeit von der optischen Drehung' war hier zu erwarten, daß3 auch die optische Drehung durch Natriumacetat heraufgesetzt werden

1) R. Feulgen, Diese Zeitschr., Bd. 91, S. 169. 
kann. Dies ist in der Tat der Fall, wie nachstehender Versuch zeigt.

$0,40 S 7 \mathrm{~g}$ nucl. Natr. in Wasser zu $10 \mathrm{ccm}$ gelöst. $1=1, t=23^{\circ}$, $c=4,09 ; \alpha=2,68^{\circ}$.

$0,4070 \mathrm{~g} \mathrm{nucl}$. Natr. in $10 \%$ iger Natriumacetatlösung zu $10 \mathrm{ccm}$ gelöst. $\mathrm{I}=1, \mathrm{t}=23^{\circ}, \mathrm{c}=4,07, \alpha=3,09^{\circ}$.

Die spezifischen Drehungen betragen mithin:

a) in Wasser $[\alpha]_{\mathrm{D}}^{23^{\circ}}=65,6^{\circ}$

b) in Natriumacetatlösung $[\alpha]_{D}^{230}=76,0^{\circ}$.

Auch diese Erscheinung widerspricht der entwickelten Theorie, wonach mit der Umlagerung auch eine Änderung des Dissoziationszustandes einhergehen - müsse. Natriumacetat ist in bezug auf die schwachen Säuregruppen kein Elektrolyt mit gleichem Ion, ein erhebliches Rückgängigwerden des Dissoziationsgrades der Säuregruppen ist also durch Natriumacetat nicht zu erwarten. Außerdem sind die freien Säuregruppen wegen ihrer Schwäche in einem derartig geringen Dissoziationszustande, daß ein weiteres Verringern dieses Zustandes optisch nicht zu bemerken wäre. Dennoch ist eine erhebliche Heraufsetzung der spezifischen Drehung und der Gelatinierfähigkeit durch Natriumacetat zu beobachten.

III. Die außergewöhnlich große Abhängigkeit der spezifischen Drehung von der Temperatur.

Es zeigte sich, daß die spezifische Drehung und damit auch der Gallertzustand in außerordentlichem Maße beeinflußbar ist durch die Temperatur, und zwar bewirkt Temperaturerhöhung einen Abfall der spezifischen Drehung und eine Verminderung der Gelatinierfähigkeit bis zu fast völligen Verschwinden der spezifischen Drehung, die bei $100^{\circ}$ nur noch wenige Grade beträgt. Auch diese Erscheinung kann nicht aus dem Dissoziationszustande der Säuregruppen erklärt werden. Zwar wird durch Temperatursteigerungen der Dissoziationszustand von Säuren erhöht, aber nur in geringen Grenzen. Wenn aber hier die Abnahme der optischen Drehung Hand in Hand gehen würde mit der Zunahme der elektrolytischen Dissoziation, so müßten bei $100^{\circ}$, bei welcher Temperatur die 
spezifische Drehung nur wenige Grade beträgt, die Säuregruppen sich in fast völlig dissọziiertem Zustande befinden, die Säuregruppen müßten dann bei hoher Temperatur hinsichtlich ihrer Stärke den Charakter von starken Mineralsäuren haben, was natürlich ausgeschlossen ist.

Man könnte hier einwenden, die Abnahme der spezifischen Drehung bei steigender Temperatur sei eine bei vielen Substanzen beobachtete Erscheinung und brauche garnichts Spezifisches zu sein, ganz besonders brauche sie nicht der Ausdruck für die oft besprochene innere Umlagerung zu sein.

Da dieser Einwand berechtigt ist, so mußte erst untersucht werden, ob der beim Erhitzen beobachtete Absturz der Rotation überhaupt der Ausdruck für die Umlagerung innerhalb der kohlenhydratähnlichen Komplexe ist oder eine $\mathrm{Zu}$ fallserscheinung. In der Gelatinierfähigkeit haben wir nun aber ein zuverlässiges Mittel kennen gelernt, die innere Umlagerung äußerlich zu erkennen; wenn sich also auch bei den Erscheinungen, wie sie beim Erhitzen auftreten, eine enge Beziehung zwischen optischer Drehung und Gallertzustand ergeben sollte, so wäre es sehr wahrscheinlich, daß auch hier die drei Dinge: Innere Umlagerung, optische Drehung und Gelatinierfähigkeit, voneinander abhängig sind. Die Beziehungen zwischen optischer Drehkraft und Gallertzustand sind, wie gezeigt werden wird, gerade beim Erhitzen so überraschend, $\mathrm{da} \beta$ an einer engen Abhängigkeit dieser beiden physikalischen Erscheinungen nicht gezweifelt werden kann.

Die Untersuchungen selbst wurden so ausgeführt, daß bei einer ungefähren Konzentration von $10 \mathrm{~g}$ in $100 \mathrm{ccm}$ die spezifischen Drehungen bei steigender Temperatur (von 5-100") beobachtet wurden. Die Änderungen, die sich hinsichtlich der Konzentration bei verschiedenen Temperaturen ergaben, wurden unberücksichtigt gelassen, weil sie in Anbetracht der großen Verschiebungen, die in den Werten der spezifischen Drehung auftraten, nur geringfügig sind. Beim Abkühlen der im Beobachtungsrohr erhitzten Lösung von nucleinsaurem Natrium wurden die Werte bei den während des Anstieges gewählten Temperaturen kontrolliert: Es ergab sich, daß die 
in der Hitze aufgetretene fast völlige Inaktivierung reversibel war, und zwar trat die Drehkraft in genau der gleichen Weise beim Abkühlen wieder auf, wie sie beim Erwärmen abgenommen hatte; denn bei gleichen Temperaturen wurden gleiche spezifische Drehungen ermittelt.

Die im einzelnen beobachteten Werte waren folgende:

$$
1=1 ; c=9,59 \text {. }
$$

\begin{tabular}{c|c|c}
\hline \hline Temperatur & $\alpha$ & {$[a]_{\mathrm{D}}$} \\
\hline $7^{\circ}$ & $6,89^{\circ}$ & $71,8^{\circ}$ \\
$10^{\circ}$ & $6,74^{\circ}$ & $70,3^{\circ}$ \\
$15^{\circ}$ & $6,39^{\circ}$ & $66,6^{\circ}$ \\
$20^{\circ}$ & $6,01^{\circ}$ & $62,7^{\circ}$ \\
$25^{\circ}$ & $5,24^{\circ}$ & $54,6^{\circ}$ \\
$30^{\circ}$ & $4,64^{\circ}$ & $48,4^{\circ}$ \\
$35^{\circ}$ & $3,89^{\circ}$ & $40,5^{\circ}$ \\
$40^{\circ}$ & $3,14^{\circ}$ & $32,7^{\circ}$ \\
$45^{\circ}$ & $2,64^{\circ}$ & $27,5^{\circ}$ \\
$50^{\circ}$ & $2,14^{\circ}$ & $22,3^{\circ}$ \\
$60^{\circ}$ & $1,64^{\circ}$ & $17,1^{\circ}$ \\
$70^{\circ}$ & $1,14^{\circ}$ & $11,9^{\circ}$ \\
$80^{\circ}$ & $0,89^{\circ}$ & $9,3^{\circ}$ \\
$90^{\circ}$ & $0,74^{\circ}$ & $7,7^{\circ}$ \\
$100^{\circ}$ & $0,64^{\circ}$ & $6,7^{\circ}$
\end{tabular}

Wie man sieht, übertreffen die Änderungen, wie sie hier bei Temperaturunterschieden beobachtet wurden, bei weitem die bei aktiven Substanzen sonst üblichen Schwankungen in der spezifischen Drehung. Um die Werte besser vergleichen zu können, wurden sie in einer Kurve zum Ausdruck gebracht, deren Abszissen die Temperaturen, deren Ordinaten die bei diesen Temperaturen beobachteten spezifischen Drehungen darstellen. Man erhält so die Kurve der Fig. 2.

Zunächst muß man feststellen, daß die Kurve nicht, wie die der Fig. 1, eine gerade Linie darstellt, der Vorgang also kein proportionaler ist. Vielmehr tritt in der Abnahme der spezifischen Drehungen in der Nähe des Siedepunktes des Wassers ein Gleichgewichtszustand ein, der auch durch weitere 
Temperatursteigerungen nicht mehr wesentlich beeinflußt werden kann.

Nun sollte, wie erinnerlich, der Nachweis geführt werden, daß der Abfall der spezifischen Drehung auch wirklich der

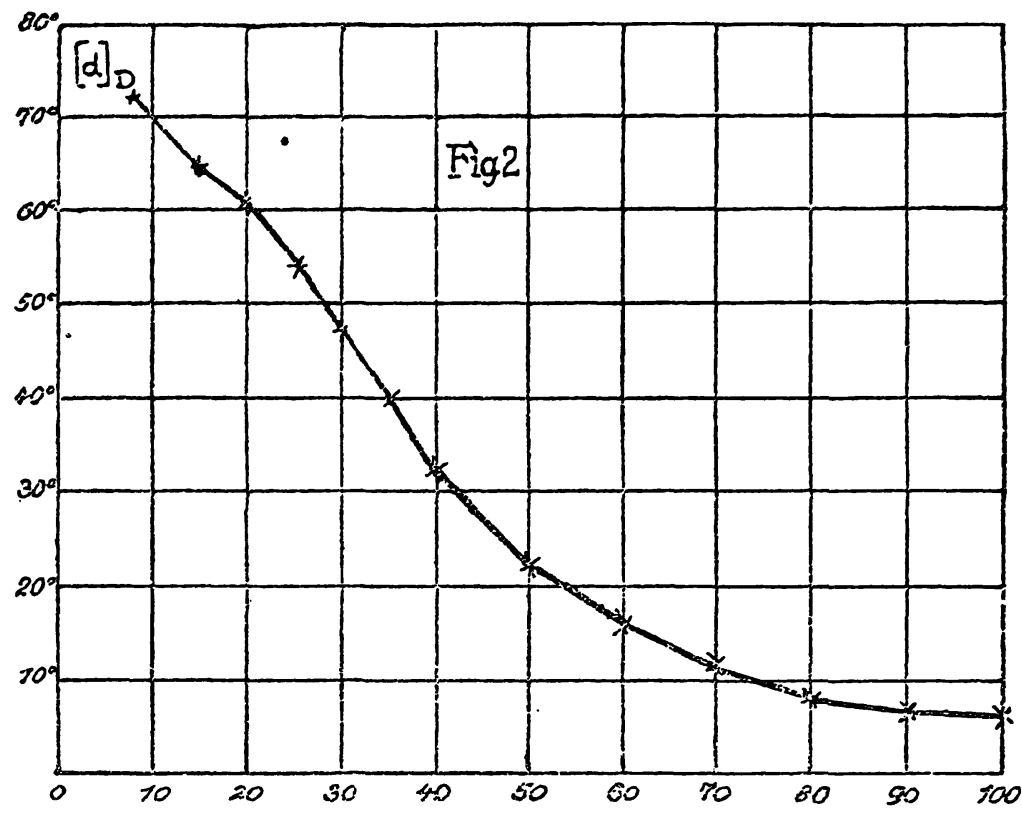

Ausdruck für die besprochene innere Umlagerung in den kohlenhydratähnlichen Komplexen ist. Wenn das der Fall ist, so mußste auf Grund unserer früheren Erfahrungen auch eine entsprechende Abnahme der Gelatinierfähigkeit zu beobachten sein. Dies ist in der Tat der Fall. Die Gallerte wird bei höherer Temperatur weicher. Führt man die Beziehungen zwischen Umlagerung, optischer Drehung und Gelatinierfähigkeit bis zur letzten Konsequenz durch, so kommt man zu dem Schlusse, daß bei der Temperatur, bei der das Drehvermögen gleich 0 wird, auch die Gelatinierfähigkeit gleich 0 sein muß: Mithin muß diese Temperatur der theoretische Schmelzpunkt der Gallerte von a-nucleinsaurem Natrium bei der der optischen Beobachtung zugrunde gelegten Konzentration sein. Nun wird aber die optische Drehung auch bei $100^{\circ}$ nicht völlig 0 , sondern bleibt, wenn auch in geringem 
Betrage (ca. $7^{\circ}$ ) bestehen, der sich bei weiterer Temperatursteigerung nur unwesentlich ändert. Dies deutet offenbar an, daß wirklich unversehrtes a-nucleinsaures Natrium in der beobachteten Konzentration c = 10 gar keinen richtigen Schmelzpunkt hat, sondern daß vielmehr bei $100^{\circ}$ bestenfalls eine Erweichung bis vielleicht zur Dickflüssigkeit zu beobachten ist, die sich bei Temperaturen um $100^{\circ}$ nicht mehr wesentlich ändern kann.

Die experimentelle endgültige Klärung dieser Frage ist natürlich abhängig von der Darstellbarkeit eines wirklich unversehrten a-nucleinsauren Natriums von maximaler Gelatinierfähigkeit.

Die Darstellbarkeit eines solchen Präparates ist nun keineswegs eine Selbstverständlichkeit. Denn sowohl nach dem Neumannschen Verfahren ') als auch nach der von mir $^{2}$ ) modifizierten Methode wird ein halbstündiges Kochen der Thymusdrüsenmasse mit Natronlauge gefordert. Nun besteht gar kein Zweifel, daß durch dieses Erhitzen mit Natronlauge ein Teil des a-nucleinsauren Natriums in die nicht gelatinierende b-Form umgewandelt wird, wenn nichts Schlimmeres (Zersetzung der Nucleinsäure) eintritt. Infolgedessen erhält man mit solchen Präparaten nicht die theoretisch geforderten hohen Schmelzpunkte. Würde man auf das halbstündige Kochen mit Natronlauge verzichten, so würde das Eiweiß nicht genügend denaturiert werden, und nach dem Neutralisieren mit Essigsäure und Fällen mit Alkohol erhielte man nicht nucleinsaures Natrium, sondern unlösliches nucleinsaures Eiweiß\}.

Ich habe nun eine Methode ausgearbeitet, mit der diese Schwierigkeiten überwunden werden können. Eine genaue Beschreibung soll einer späteren Arbeit vorbehalten bleiben; hier will ich nur auf das Prinzip eingehen.

Die zerkleinerte Drüsenmasse wurde in Wasser bei $95^{\circ}$ suspendiert und die Natronlauge zugegeben. Der größte Teil der Masse löste sich momentan und das Gelöste wurde von dem Ungelösten in wenigen Augenblicken mittels Durchseihens

1) Archir für Physiologie 1899, Suppl., S. 553.

2) Diese Zeitschr., Bd. 90, S. 261.

Hoppo-Seyler's Zeitschrift 1. physiol. Chemie. CrV. 
darch ein Sieb getrennt. Würde man nun nach der alten Methode mit Essigsäure neutralisieren und mit Alkohol fällen, so orhielte man, wie gesagt, kein nucleinsaures Natrium, sondern unlösliches nucleinsaures Eiweiß. Es zeigte sich aber, daß, wenn man aus alkalischer Lösung mit Alkohol fällte, dann das Eiweiß aus der Salzbindung mit der Nucleinsäure ferngehalten wurde. Würde man aber die alkalische verflüssigte Drüsenmasse ohne weiteres mit Alkohol zu fällen versuchen, so würde man gar keinen Niederschlag von nucleinsaurem Natrium erhalten; denn es würde ja das inaktive Salz entstehen, und dieses wäre wegen seiner schwierigen Fällbarkeit aus der alkalischen Eiweißlösung gar nicht als faßbarer Niederschlag zu bekommen. Versetzt man nun aber die alkalische lösung mit einer der Natronlauge äquivalenten Menge Ammoniumchlorid, so bildet sich, wie eingangs auseinandergesetzt, sofort die leicht fällbare aktive Modifikation, während das an die Stelle der Natronlauge getretene Ammoniak noch eine genügend starke Base ist, um das Eiweiß aus der Salzbindung mit der Nucleinsäure abzuhalten.

Versetzt man also nach der auf Natronlaugezusatz augenblicklich eingetretenen Verflüssigung sofort mit Alkohol und Ammoniumchlorid, so erhält man, wie man sieht, ein a-nucleinsaures Natrium zunächst als Rohprodukt, das tatsächlich nur momentan der Natronlaugenwirkung ausgesetzt war.

Derartig dargestellte Präparate wurden nun hinsichtlich des Verhaltens ihrer Gallerten geprüft, und zwar wurde, entsprechend der Konzentration bei den optischen Versuchen, auch hier eine Konzentration von annähernd $10 \mathrm{~g}$ auf $100 \mathrm{ccm}$ gewählt; doch konnte die Lösung wegen zu intensiver Gallertbildung nicht im Meßkölbchen hergestellt werden, sondern nur gravimetrisch, unter Vernachlässigung des spezifischen Gewichtes. Schon beim Übergießen des a-nucleinsauren Natriums mit Wasser zeigte sich die hohe Gelatinierkraft dieses Präparates. Es bildete sich eine feste Masse, die selbst in einer Menge von $5 \mathrm{~g}$ im Reagensglase kaum durchgerührt werden konnte. Beim Erhitzen im siedenden Wasserbade konnte die Masse erst nach 10 Minuten langem Verweilen im Wasser- 
bade in Fluß gebracht werden. Sie wurde im maximal erweichten Zustande durch Neigung des Rohres an der Wandung ausgebreitet und nach Art der in der bakteriologischen Praxis üblichen schrägen Kulturröhrchen erstarren gelassen. Die entstandene Gallerte hatte die Konsistenz von Gummi oder gutem erstarrten Tischlerleim. Das Reagensglas wurde nun in einem Becherglase mit Wasser steigenden T'emperaturen ausgesetzt, und da zeigte sich in der Tat das aus der Kurve 2 vorhergesagte Verhalten: Ein eigentlicher Schmelzpunkt war garnicht zu beobachten. Erst bei etwa $80^{\circ}$ (!) fing die inzwischen weicher gewordene Gallerte an, am Glase herunterzulaufen. Dies vollzog sich sehr langsam, und bei $90^{\circ}$ war das Herunterlaufen noch nicht beendet. Die Masse stellte auch bei $100^{\circ}$ keine eigentliche Lösung dar, sondern war vielmehr so viskös, daß eine Filtration selbst im siedenden Heißwassertrichter gänzlich ausgeschlossen war. Das Verhalten der Gallerte steht also in vollkommener Übereinstimmung mit den aus der Kurve 2 erwarteten Eigenschaften, und man muß auch den beim Erhitzen beobachteten Absturz der spezifischen Drehung zurückführen auf jene innere Umwandlung, die auch in der Änderung des Gallertzustandes zum Ausdruck kommt, und die sich also auch hier wieder vollziehen kann, ohne daß der Dissoziationsgrad der schwachen Säuregruppen sich wesentlich änderte.

Der Vollständigkeit halber sei hier auch die Abhängigkeit der spezifischen Drehung von der Konzentration zahlenmäßjig wiedergegeben. Sie ist sehr erheblich, und die spezifischen Drehungen nehmen mit steigender Verdünnung ab. In unserem speziellen Falle hat dieser Vorgang deswegen weniger Interesse, weil sich hier die Abhängigkeit der optischen Drehung von der Gelatinierfähigkeit nicht ohne weiteres nachweisen läßt, da es an einer brauchbaren Methode fehlt, die Gelatinierfähigkeit bei verschiedenen Konzentrationen (also unabhängig von der Konzentration) zu vergleichen.

$$
1=1 \text {; }
$$

$1,5 \mathrm{~g} \mathrm{zu} 10 \mathrm{ccm}$ gelöst: $\mathrm{c}=12,15 ; \alpha=9,28{ }^{\circ} ;[\alpha]_{\mathrm{D}}^{23^{\circ}}=76,3^{\circ}$

$$
1,5, \Rightarrow 25, \quad, \quad c=4,86 ; \alpha=2,590^{\circ} ;[\alpha]_{\mathrm{D}}^{23^{\circ}}=53,3^{\circ}
$$


$1,5 \mathrm{~g} \mathrm{zu} 50 \mathrm{ccm}$ gelöst: $\mathrm{c}=2,43 ; \%=1,09^{\circ} ;[\alpha]_{\mathrm{D}}^{23^{\circ}}=44,8^{\circ}$ $1,5,100, \quad: c=1,215 ; \alpha=1,215^{\circ} ;[\sigma]_{\mathrm{D}}^{23^{\circ}}=40,3^{\circ}$.

Man erkennt, daß die spezifischen Drehungen mit größerer Verdünnung bis auf die Hälfte abnehmen können. Höchst wahrscheinlich ist damit auch eine entsprechende Abnahme der Gelatinierfähigkeit verbunden. Da diese auch außerdem lediglich wegen der Verdünnung unter allen Umständen eintritt, so haben wir in der Verdünnung ein Mittel in der Hand, um die beim Arbeiten geradezu unerträgliche Gelatinierfähigkeit erheblich herabzusetzen. So gelingt es denn auch, in sehr starker Verdünnung $(1 \%)$, selbst das völlig intakte und hochgelatinierende a-nucleinsaure Natrium wenigstens in kleinen Mengen im Heißwassertrichter zu filtrieren.

Ich habe eine Reihe von Erscheinungen besprochen, die darauf hindeuteten, daß die innere Umlagerung, verbunden mit einer Änderung der optischen Eigenschaften und des Gallertzustandes, vollzogen werden kann, ohne daß der Dissoziationsgrad der schwachen Säuregruppen eine Änderung erführe, daß mithin eine Herabsetzung der Aktivität nicht unbedingt an eine Salzbildung gebunden ist, wohl aber durch eine solche herbeigeführt wird.

Eine Herabsetzung bezw. Aufhebung der Aktivität kann erreicht werden:

1. Durch Alkalien, unter Bildung der Salze. Der Prozeß verläuft bei starken Alkalien quantitativ und proportional (geradliniger Verlauf der Kurve 1).

2. Durch Säuren. Sie dürfen nicht stärker als Nucleinsäure sein, weil diese sonst ausgefällt werden würde. Der Prozeß verläuft nicht quantitativ, aber zum Teil, und es entstehen die freien Säuregruppen.

3. Durch Temperatursteigerungen. Bei hoher Temperatur findet die Inaktivierung fast ganz, aber nicht restlos statt, daher auch keine vollständige Verflüssigung der Gallerte. Dieser Prozeß ist ein umkehrbarer und vollzieht sich beim Abkühlen in entgegengesetzter Richtung. 
Alle diese Beobachtungen legten mir den Gedanken nahe, daß die schwachen Säuregruppen in den aktiven Molekülen nicht frei, sondern in Form von Bindungen vorkommen, die durch Säuren, ganz besonders glatt aber durch Alkalien unter Bildung der Salze gelöst werden. Im geöffneten Zustande der Bindungen ist das Molekül der Nucleinsäure inaktiv. Auch Temperatursteigerungen würden im Sinne einer Aufspaltung und deswegen inaktivierend wirken. Es besteht große Nèigung zum Schlusse der Bindungen; dieser vollzieht sich deshalb wieder, wenn die spaltende Ursache beseitigt ist.

Wenn aber eine reversible Inaktivierung durch Temperatursteigerung geschehen kann, so folgt daraus, $d a ß$ in einer Lösung von nucleinsaurem Natrium auch bei Zimmertemperatur keineswegs nur aktive Moleküle bestehen, daß vielmehr auch inaktive Moleküle vorhanden sind. Mit anderen Worten, es besteht $\mathrm{zwischen}$ den inaktiven und aktiven Molekülen ein Gleichgewichtszustand, der abhängig ist von der Temperatur. Verschieben läßt sich dieser Gleichgewichtszustand zugunsten der aktiven Moleküle durch Zusatz von Salzen (Natriumacetat), Abkühlung oder größere Konzentration, durch welche Maßnahmen infolgedessen auch die Gelatinierfähigkeit erhöht wird. Sind die Säuregruppen einmal mittels Alkalien in stabile, inaktive Salzform gebracht, so ist eine Schließung der Bindungen, das Auftreten aktiver Moleküle im Salzzustande natürlich ausgeschlossen; d. h. das durch Alkali vollständig inaktivierte nucleinsaure Natrium ist hinsichtlich seines inaktiven Zustandes und seiner mangelnden Gelatinierfähigkeit unabhängig von der Konzentration, Temperatur und Salzgehalt, was ja auch durch den Versuch bestätigt wird.

Die Inaktivierung des nucleinsauren Natriums durch Alkalien ist etwas so Charakteristisches, daß man dieses Verhalten geradezu als ein bequemes Erkennungsmittel für Nucleinsäuren vom Bau der echten Nucleinsäure benutzen kann, und man muß die Nucleinsäuren einteilen in solche, die mit Alkali inaktiviert werden können, und solche, bei denen 
210 R. Feulgen, Ein optisch-inaktives Natriumsalz der Nucleinsäure.

dies nicht möglich ist. Zur letzteren Gruppe gehört z. B. die Guanylsäure, deren schwache Linksdrehung auch in Gegenwart von starken Alkalien bestehen bleibt. Von Interesse wird also sein, auch andere Nucleinsäuren auf dieses Verhalten hin zu untersuchen.

Zum Schluß sei auf eine eigenartige Verwendungsmöglichkeit des inaktiven nucleinsauren Natriums aufmerksam gemacht. Man kann es als Reagens auf schwache Säuregruppen oder auf als Säure wirkende Gruppen verwenden. Man würde. dabei so verfahren, daß man eine Lösung von nucleinsaurem Natrium mit Natronlauge bis fast zum Verschwinden jeglichen Drehvermögens versetzt, eine äquivalente Menge der zu untersuchenden Substanz hinzufügt und polarimetrisch beobachtet: Tritt Drehung auf, so sind Säuregruppen vorhanden, die stärker als die Säuregruppen in der Nucleinsäure sind. Es lassen sich so noch Säuregruppen erkennen, die mit Indikatoren nicht mehr nachgewiesen werden können.

Die Untersuchungen wurden mit Mitteln aus der "Gräfin Bose-Stiftung" ausgeführt. 

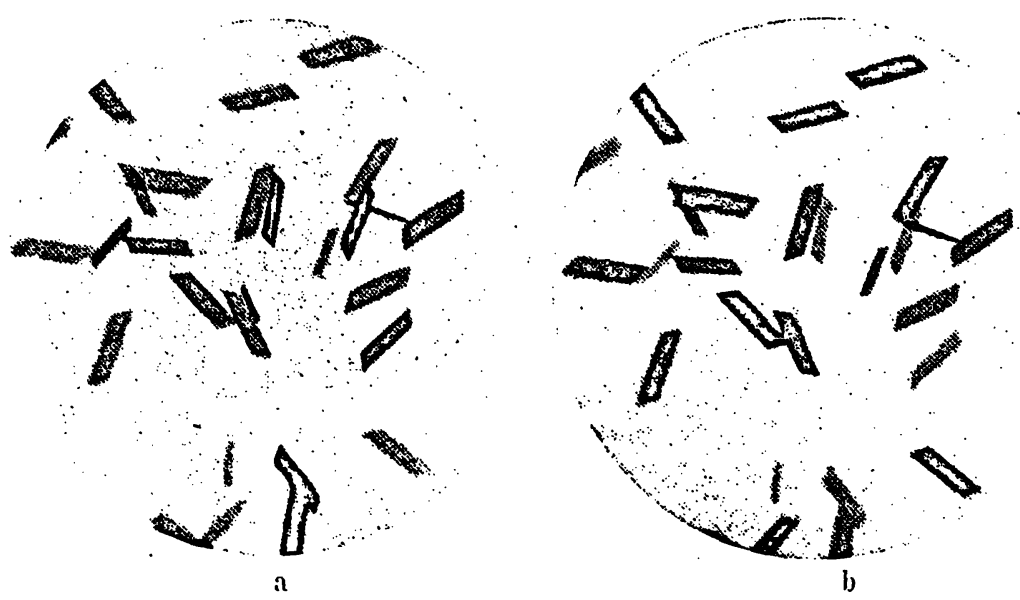

ligg. 1. Teichmannsche Hüminkristalle aus Meerschweinchenblut. Cl- und J-haltig. Aufnahmen in polarisiertem Licht.

a) Normalstellung des Nikol, b) um $90^{\circ}$ gegen a) gedreht. Aufgenounmen mit. Zeiss Öl-Immersion 2,0 mm, Ok. \&, Balgl. $280 \mathrm{~mm}$, Vorgr. 250,0.

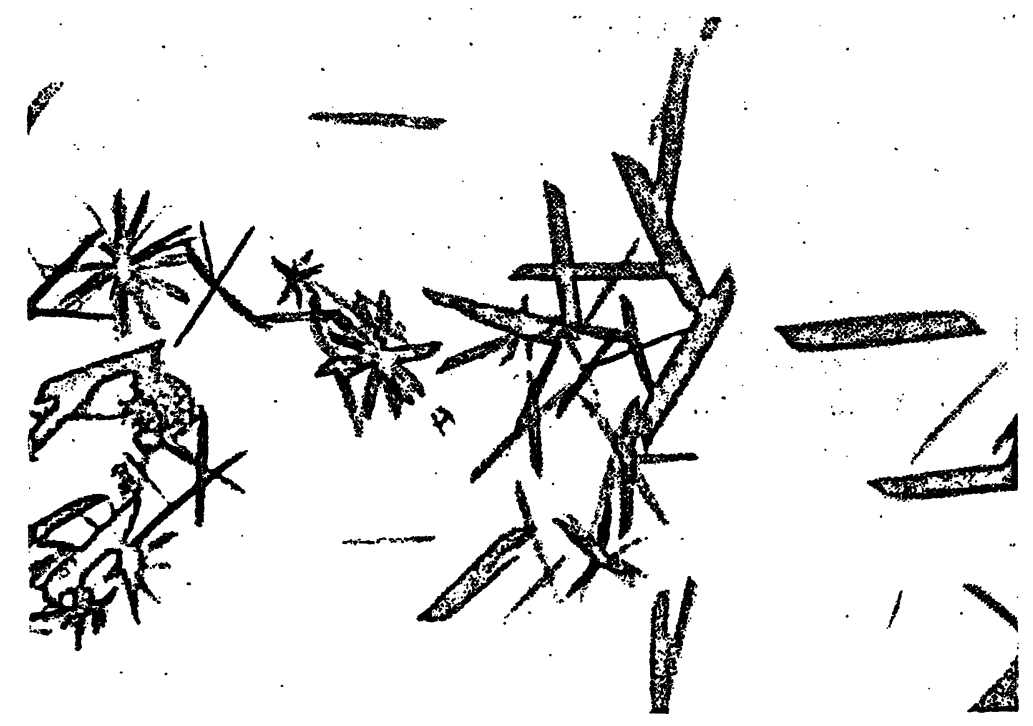

Fig. 2. Schalfeje wsche Häminkristalle aus Rinderblut. $\mathrm{Cl}$ - und $\mathrm{Br}$-haltig. Aufgenonmen mit Himmler Cbj. 7, Ok. 4. Balgl. 240 mrn. Vergr. 432,0.

-Hoppe-Seyler's Zeitschrift f. physiol. Chemic- Bd. 104, Tufel 1. $\mathrm{Zu}{ }_{7} \mathrm{H}$ intzelmanu, Nachweis ron Jod etc.". 
Brought to you by | Purdue University Libraries Authenticated

Download Date | 5/23/15 4:34 AM 